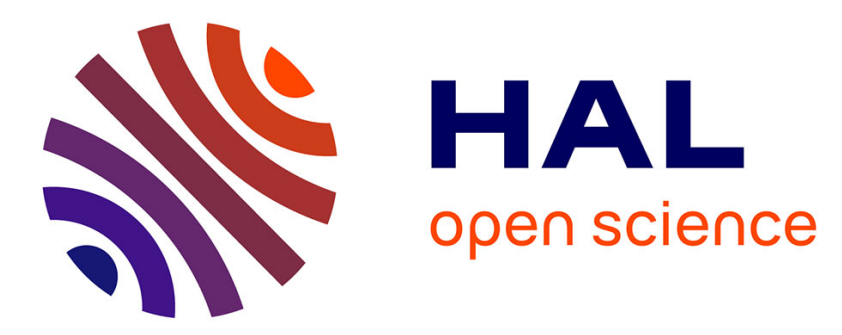

\title{
Nonlinear acoustic measurements to assess crack density in trabecular bone
}

\author{
Guillaume Renaud, Samuel Callé, Jean-Pierre Remenieras, Marielle
}

Defontaine

\section{- To cite this version:}

Guillaume Renaud, Samuel Callé, Jean-Pierre Remenieras, Marielle Defontaine. Nonlinear acoustic measurements to assess crack density in trabecular bone. International Journal of Non-Linear Mechanics, 2008, 43 (3), pp.194. 10.1016/j.ijnonlinmec.2007.12.007 . hal-00501767

\section{HAL Id: hal-00501767 https://hal.science/hal-00501767}

Submitted on 12 Jul 2010

HAL is a multi-disciplinary open access archive for the deposit and dissemination of scientific research documents, whether they are published or not. The documents may come from teaching and research institutions in France or abroad, or from public or private research centers.
L'archive ouverte pluridisciplinaire HAL, est destinée au dépôt et à la diffusion de documents scientifiques de niveau recherche, publiés ou non, émanant des établissements d'enseignement et de recherche français ou étrangers, des laboratoires publics ou privés. 


\section{Author's Accepted Manuscript}

-

NON-LINEAR

MECHANICS

Nonlinear acoustic measurements to assess crack density in trabecular bone

Guillaume Renaud, Samuel Callé, Jean-Pierre Remenieras, Marielle Defontaine

PII: S0020-7462(07)00235-1

DOI: doi:10.1016/j.ijnonlinmec.2007.12.007

Reference: NLM 1429

To appear in: International Journal of NonLinear Mechanics

Received date: 25 May 2007

Revised date: 12 October 2007

Accepted date: 18 December 2007

Cite this article as: Guillaume Renaud, Samuel Callé, Jean-Pierre Remenieras and Marielle Defontaine, Nonlinear acoustic measurements to assess crack density in trabecular bone, International Journal of Non-Linear Mechanics (2007), doi:10.1016/j.ijnonlinmec.2007.12.007

This is a PDF file of an unedited manuscript that has been accepted for publication. As a service to our customers we are providing this early version of the manuscript. The manuscript will undergo copyediting, typesetting, and review of the resulting galley proof before it is published in its final citable form. Please note that during the production process errors may be discovered which could affect the content, and all legal disclaimers that apply to the journal pertain. 


\title{
Nonlinear acoustic measurements to assess crack density in trabecular bone
}

\author{
Guillaume Renaud*, Samuel Callé, Jean-Pierre Remenieras, \\ Marielle Defontaine \\ Université François Rabelais, Laboratoire UltraSons Signaux et Instrumentation, \\ CNRS FRE 2448, 10 Boulevard Tonnelle, 37032 Tours cedex 1, France
}

\begin{abstract}
Three methods to measure qualitative and quantitative aspects of bone nonlinearity were investigated in the context of diagnosis of bone "biomechanical health": i.e. harmonic generation, parametric emission and parametric reception using phase modulation. Trabecular bone exhibited hysteretic nonlinear behavior due to microcracks in bone tissue, and parametric reception using phase modulation seemed to be the best configuration for in vivo application. However, the relationship between level of nonlinearity and crack density needs to be validated by histological analysis.
\end{abstract}

Key words: Acoustic waves interactions, Harmonic generation, Nonlinear elasticity, Microdamage, Bone PACS: 43.25.Ba, 43.25.Zx, 43.80.Qf

\section{Introduction}

It is possible in the skeleton to distinguish between cortical bone with low porosity (less than 30\%) and trabecular bone with high porosity (more than $70 \%$ ). As most bone fractures in the elderly occur in trabecular bone, this study focused on this type of bone, particularly the heel bone. Trabecular bone is a spongy bi-phasic material with connected porosity. Its solid phase consists of a connected network of bone tissue rods known as trabeculae. Porous spaces are filled with marrow (mostly fat). The clinical diagnosis of bone fragility is currently performed using X-ray measurements which provide imaging of bone mineral density. This quantitative assessment, similar

* guillaume.renaud28@etu.univ-tours.fr 
to porosity measurement, accounts for $60-70 \%$ of variation in bone strength [1]. However measuring the amount of material in a porous structure is not enough to evaluate its strength. It also depends on the spatial arrangement of the material and on the mechanical characteristics of the bone tissue. Furthermore, it is well known that cracks typically of tens to hundreds of $\mu \mathrm{m}$ in size are generated in bone tissue by daily loading. Figure 1 shows different types of common bone microdamage. In healthy bone, damage is repaired by continuous bone remodeling. When an imbalance in this process occurs, cracks can accumulate.

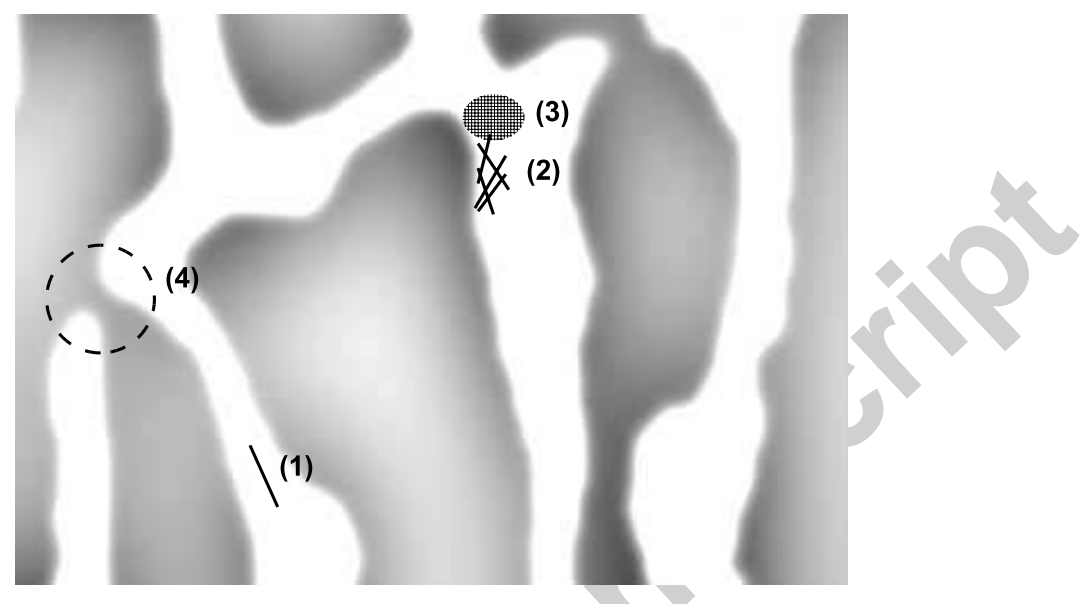

Fig. 1. Four types of damage in trabecular bone (grey areas represent marrow and white areas represent bone tissue): (1) isolated crack, (2) cross-hatched cracks, (3) diffuse damage and (4) broken trabeculae. The field of view is about $1.5 \mathrm{~mm}$ wide.

Crack surface density, measured by histological analysis using staining techniques [3], and the associated standard deviation increase with age [8] [13] [30]. Whereas crack surface density is below 1 crack per $\mathrm{mm}^{2}$ in young healthy people, it can reach values above 10 cracks per $\mathrm{mm}^{2}$ in elderly people. Whether the increase in crack density is a cause or a consequence of the decrease in the bone toughness properties with age is still not resolved [2] [23] [31].

Linear ultrasound imaging techniques have been developed to image trabecular bone velocity and attenuation [6]. These techniques are nonetheless almost insensitive to progressive induced damage [18]. On the other hand, it is now well established that inhomogeneous materials, with soft mesoscopic flaws embedded in a rigid matrix, exhibit strong nonlinear quasi-static and dynamic behavior known as non-classical or hysteretic nonlinearity [9] [10] [20]. Non-destructive evaluation techniques are being developed to detect localized damage in materials [25] [27] [29]. Nevertheless very few studies have been performed on the relationship between crack density and level of nonlinearity [19] [25] [11] [16].

Moreover, very few studies have been undertaken on bone nonlinearity. The 
classical nonlinear quadratic parameter $\beta$ (defined as $\rho_{0} c_{0}\left(\frac{\partial c}{\partial p}\right)$, where $\rho_{0}$ is the density of the medium and $p$ the acoustic pressure) was found to range between 80-120 in bovine trabecular bone [24], whereas $\beta$ does not exceed 7.5 (PMMA) in homogeneous material [12]. Hysteretic nonlinearity has been clearly observed in bovine and human cortical bone using nonlinear resonance ultrasound spectroscopy [14] [15]. One interesting point is that the nonlinear hysteretic parameter $\alpha$, related to the resonance frequency downshift, increases overall with age as well as its dispersion. This trend is similar to the increase in crack density with aging in trabecular [8] and cortical bone [22]. Crack density is probably a mirror of the "biomechanical health" of bone tissue and the increased dispersion with age probably reveals disparities in nutrition, physical activity and diseases. Several features have been identified to improve understanding of the relationship between the level of nonlinearity and crack density. These include the effects of preferential orientation of cracks and the relative nonlinear contributions of different types of damage in bone.

We propose three different modalities to measure trabecular bone nonlinearity in this context in order to image crack density in future studies. Such noninvasive methods would be valuable for in vitro biomechanical studies, to understand how microcracks impact on the biomechanical properties of bone and for in vivo monitoring of crack accumulation (to date only possible by biopsy).

The following sections comprise a brief technical presentation and experimental results obtained from harmonic generation, parametric emission and parametric reception using phase modulation.

\section{Method 1 : Harmonic generation}

One way to investigate the nonlinearity exhibited by a material consists of increasing monofrequency excitation and observing the dependence of harmonic amplitudes on the fundamental amplitude. According to theory, in the case of weak nonlinear effects classical nonlinear materials have $\mathrm{n}^{\text {th }}$ harmonic amplitude increasing with power $n$ of the fundamental amplitude. Typical manifestation of microcracks in damaged solids is the dominance of odd harmonics and their almost quadratic dependence on pump amplitude [26].

\subsection{Experimental method}

Figure 2 describes the experimental set-up. We used a focused transducer to generate a monofrequency wave at $400 \mathrm{kHz}$ in a water tank. The width of the focused beam at $-6 \mathrm{~dB}$ was less than $1 \mathrm{~cm}$. The amplitude of the transducer 
excitation was increased from 30 to $160 \mathrm{~V}$ peak-peak. The fundamental and harmonic pressures were measured by a needle hydrophone placed at $45 \mathrm{~mm}$ along the axis of the transducer, where the fundamental, second and third harmonics reach their maximum levels in water (figure 3 ). The experiment was first performed in water without any sample. Marrow was then removed from the bone sample and the experiment was conducted with a slice of defatted heel bone (24 mm thickness) and a slice of porous ceramic (20 mm thickness) (see Table 1 for more information on the samples). The samples were placed about $20 \mathrm{~mm}$ from the transducer so that the generation of harmonics occurred only in the sample (figure 3). Because of the strong attenuation in both trabecular bone and porous ceramic, the resonance effects in the sample were expected to be very weak. Moreover, the reflection coefficient for acoustic pressure between trabecular bone (considered as an effective medium) and water is 0.1.

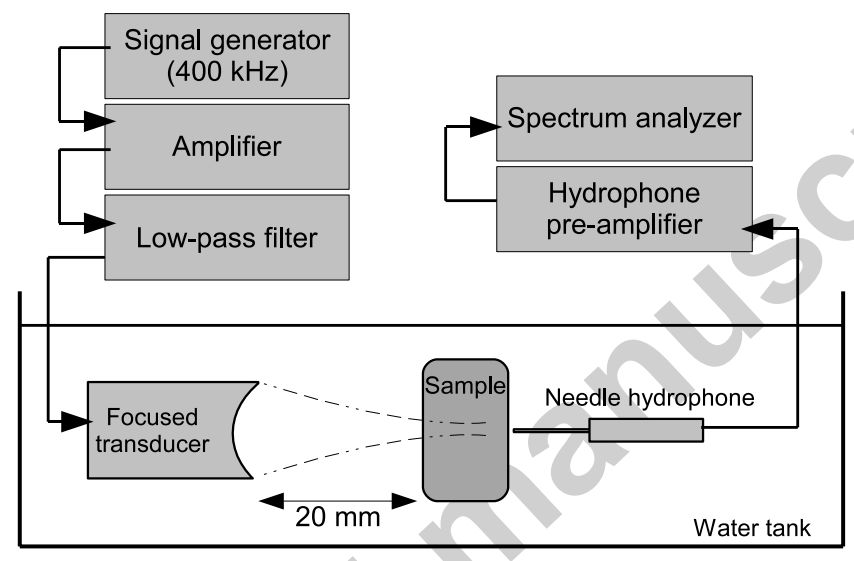

Fig. 2. Experimental configuration for harmonic generation.

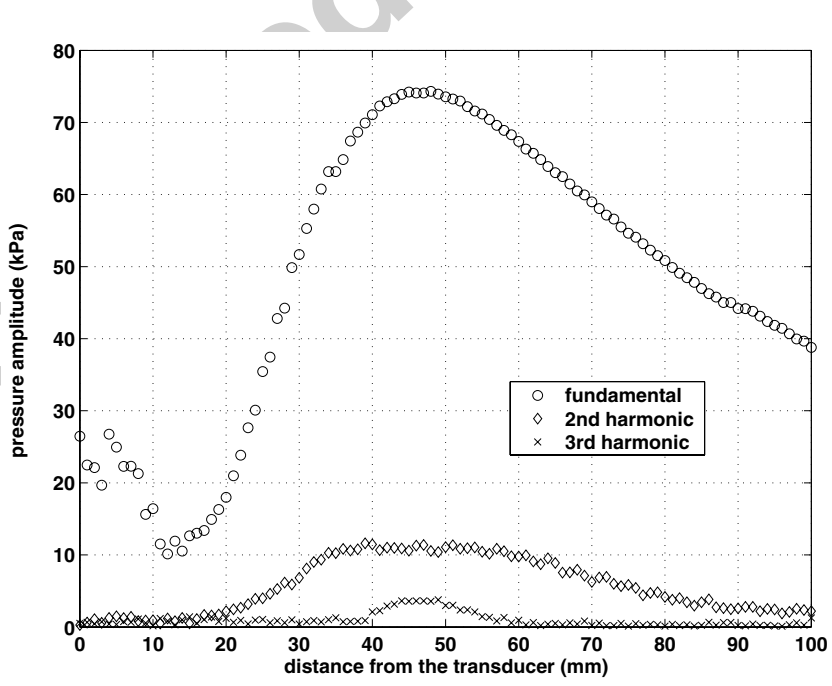

Fig. 3. Measurements of the pressure amplitude on the axis of the focused transducer in water. Amplitudes of 2 nd and 3rd harmonics are multiplied by 10 for better readability. 


\subsection{Results and interpretation}

The pressures of the fundamental, second, third and fifth harmonics measured in water, through trabecular bone and through porous ceramic are plotted as functions of the transducer voltage excitation in figures 4, 5 and 6, respectively.

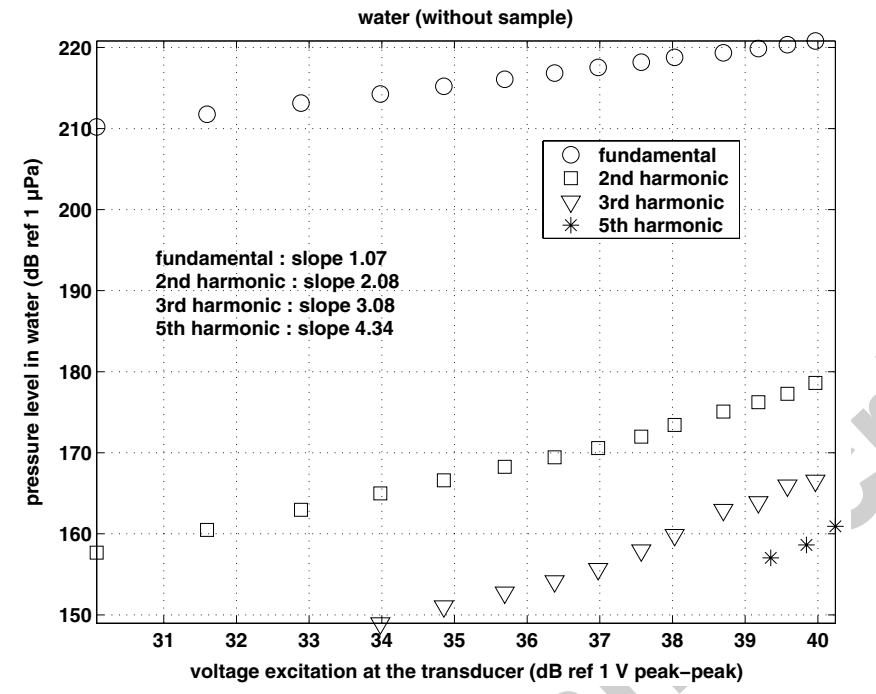

Fig. 4. Pressure levels measured in water at $45 \mathrm{~mm}$ for different levels of excitation.

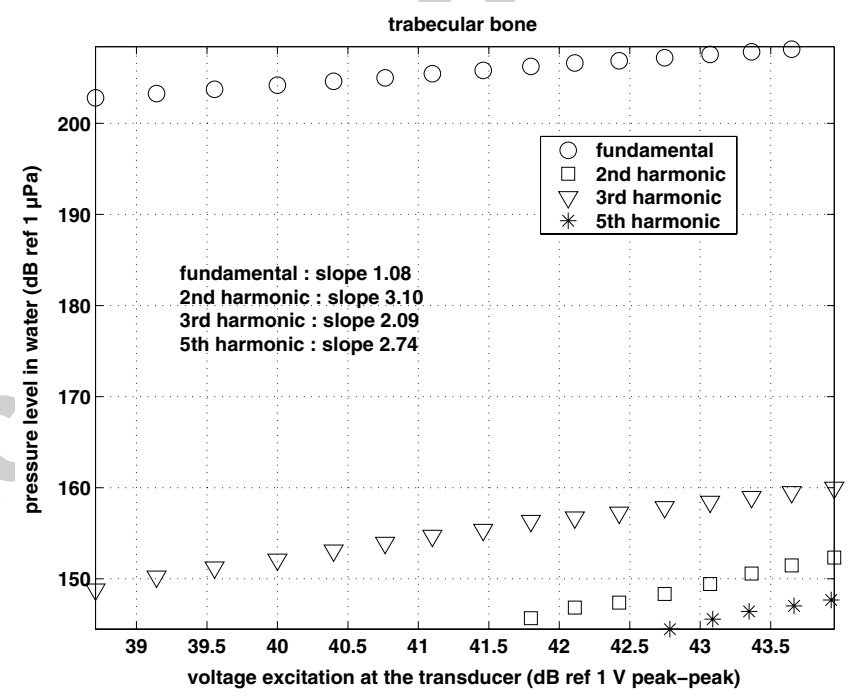

Fig. 5. Pressure levels measured in water at $45 \mathrm{~mm}$ behind the trabecular bone sample for different levels of excitation.

The maximum fundamental acoustic pressure measured in water (without 


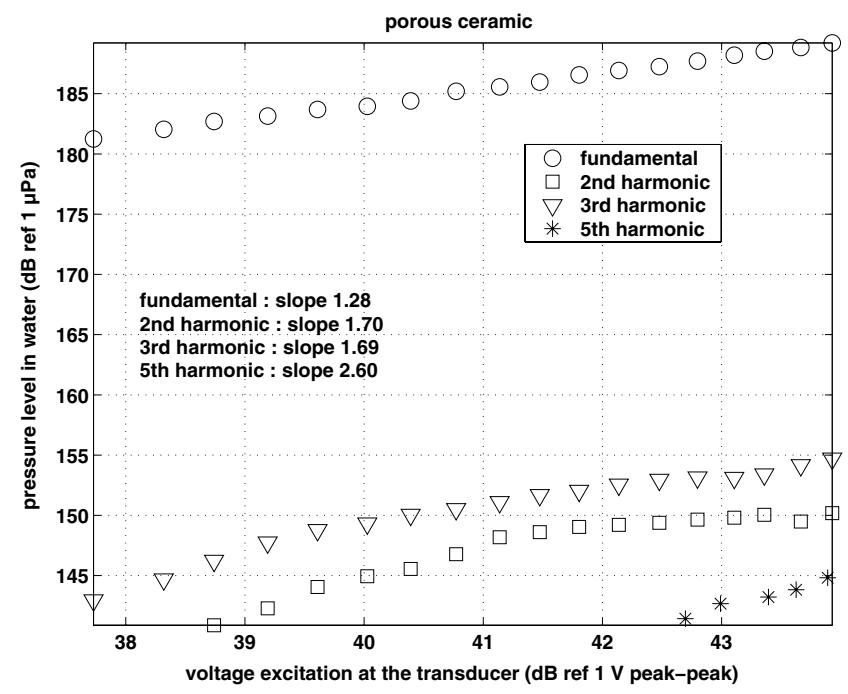

Fig. 6. Pressure levels measured in water at $45 \mathrm{~mm}$ behind the porous ceramic sample for different levels of excitation.

sample) was $112 \mathrm{kPa}$, thus the hypothesis of weak nonlinear effects is valid. The results obtained in water clearly showed classical nonlinear behaviors : second, third and fifth harmonics behaved with slopes 2.08, 3.08 and 4.34, close to the theoretical values of 2,3 and 5 , respectively. In contrast, trabecular bone and porous ceramic exhibited hysteretic nonlinearity characteristics : amplitude of the third harmonic higher than that of the second harmonic (despite very high attenuation due to scattering above $800 \mathrm{kHz}$, see Table 1) and a power law close to 2 , especially for the third and fifth harmonics. In trabecular bone the second harmonic amplitude increased with power 3 of the fundamental amplitude. This astonishing behavior was observed in damaged materials. Some studies have reported that progressively induced damage increases the power law relating the second harmonic with the fundamental amplitudes, whereas the overall level of the third harmonic was increased, without impacting on the power law [25].

The common velocity dispersion measured in trabecular bone is $-26 \mathrm{~m} / \mathrm{s} / \mathrm{MHz}$ in the frequency range $200-800 \mathrm{kHz}$ [28]. In view of the small thickness of the samples, dispersion effects may have little effect on harmonic generation.

Moreover harmonic distortion can be induced by the pressure-dependence of the flow resistivity above a certain acoustic velocity threshold. After a certain propagation distance, odd harmonics have higher amplitudes than even harmonics. For example, Kevlar with porosity of $96 \%$ exhibits that behavior when acoustic velocity exceeds $1 \mathrm{~m} / \mathrm{s}$ [17]. In the present study, the acoustic velocity did not exceed $8 \mathrm{~cm} / \mathrm{s}$ and the mean pore diameter was larger in trabecular bone $(0.5-1 \mathrm{~mm})$ than in Kevlar. We therefore assumed that this effect was negligible in our experiments. 


\section{Method 2 : Parametric emission}

An alternative way to investigate nonlinear characteristics of bone is parametric generation. We propose a method based on the combination of two focused primary beams emitted at two slightly different frequencies $\left(f_{1}\right.$ and $f_{2}$ ) using an annular array [7]. These primary waves, focused in the bone, create an amplitude modulation giving rise to a low-frequency ( $L F$ ) wave at $\mathrm{f}_{L F}$ (equivalent to the frequency difference of the two primary frequencies). An internal stress field results from this LF excitation, and the acoustic response of the object to this mechanical excitation is used to form the image. The LF acoustic field resulting from the vibration of the medium is detected by a sensitive hydrophone and then used to form the image.

Two main physical phenomena are responsible for this LF wave generation: the acoustic radiation force (due to reflection and attenuation) [7] and the nonlinear interaction [4]. The overall low frequency field $\mathrm{q}_{L F}$ can be expressed as $q_{L F}=q_{\text {rad }}+q_{N L}$, where $\mathrm{q}_{\text {rad }}$ represents the LF pressure originating from the radiation force and $\mathrm{q}_{N L}$ represents the contribution of the $\mathrm{LF}$ nonlinear interaction (parametric generation).

\subsection{Experimental configuration}

In a previous study [21] we reported that the choice of the focused annular array depends on several parameters (e.g. the curvature of the annular array, the frequency downshift ratio $\mathrm{f}_{1} / \mathrm{f}_{L F}$ and the number of rings). Finally, a $1 \mathrm{MHz}$ array with 4 rings and a focusing gain $(\mathrm{G})$ of 16.14 per element was an acceptable compromise between sensitivity and resolution for parametric generation [4]. The two odd rings emit at $\mathrm{f}_{1}$ and the two even rings emit at $\mathrm{f}_{2}$. The focal distance and the probe diameter were $6 \mathrm{~cm}$ and $8.32 \mathrm{~cm}$, respectively. In this configuration, the LF field resolution at the focus is in the same range as the primary field resolutions [5]. The LF resolution $(1 \mathrm{~mm})$ corresponds to the lateral resolution of our system, which is promising for image construction. Figure 7 presents the experimental set-up: the bone slice faces the transducer, and the LF hydrophone linked to the transducer is beside the object.

\subsection{In vitro heel bone imaging}

Due to both the overall resonance frequencies and the local bone properties, the LF pressure amplitude does not vary simply according to the choice of $\mathrm{f}_{L F}$ [5]. The optimal frequency of $76 \mathrm{kHz}$ was therefore chosen for the later heel bone images. Figure 8 represents the image of the LF pressure amplitude in the 


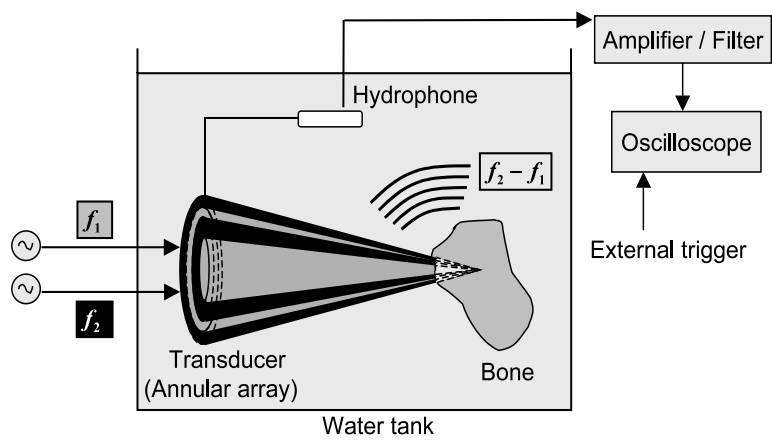

Fig. 7. Experimental set-up of the parametric emission method.

medium plane of a $19 \mathrm{~mm}$ thick human heel bone slice (figure 9). The spatial resolutions related to displacement steps were $300 \mu \mathrm{m}$ in both directions. The central frequency was $800 \mathrm{kHz}$ to minimize attenuation effects in the bone. The image clearly shows the trabeculae framework. The cortical outlines of the heel are also clearly demonstrated. Moreover, we can see that the white areas of the image (large parametric amplitude) at the bottom of the heel bone correspond to the region in contact with the ground, which undergoes daily loading. Such areas could contain many microcracks.
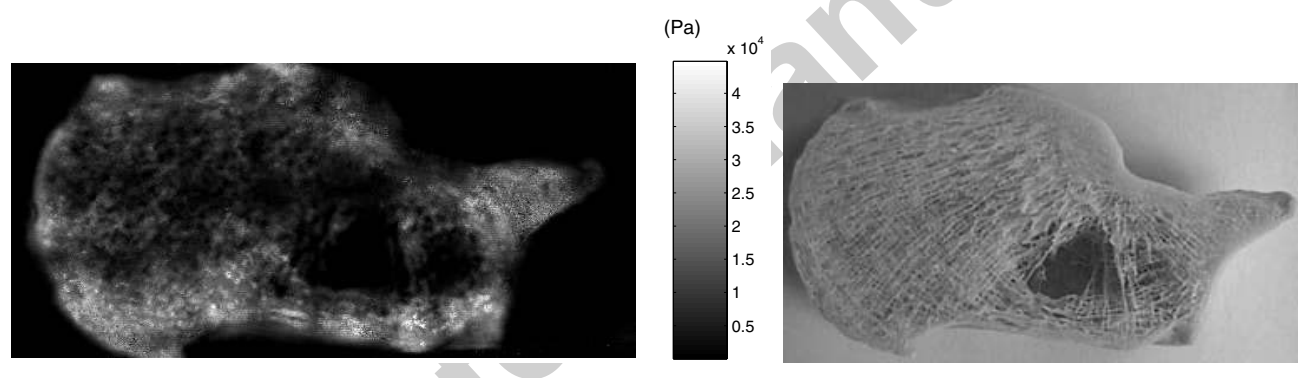

Fig. 8. Amplitude of the parametric LF wave re- Fig. 9. Photograph of the human ceived by the hydrophone. heel bone slice.

\subsection{Interpretation and discussion}

The radiation force resulting from the combined primary fields at frequencies $f_{1}$ and $f_{2}$ is one source of LF stress in the object [7]. However, we have already reported that a LF field is created by the nonlinear interference of the two primary beams inside the propagating medium [4]. This field, directly related to the coefficient of nonlinearity $\beta$, may then propagate, scatter and be reflected on the LF contrast sources of the object and thus provide a measurable radiating field. In the above study the LF acoustic response of tissue varied with tissue type and with the choice of the difference frequency, indicating that this is potentially a valuable tool for bone investigation. The 
main advantages of this method compared to other ultrasound bone imaging methods are: (i) the non-transmission mode, which for instance allows in vivo imaging of the neck of femur, and (ii) its high lateral resolution. Moreover, we reported that the creation of this internal force in our conditions depended on the attenuation and impedance (radiation force term) and the nonlinear coefficient (nonlinear interference term). In practice, the contribution of the preponderant phenomenon (radiation force or NL interaction) has not been clearly established, since it strongly depends on the type of tissue being studied and is particularly difficult to estimate in bone. Thus, although the LF signal is partly linked to the coefficient of nonlinearity $\beta$ and hence is larger for bone regions affected by microcracks, this technique does not exclusively provide information about microdamage.

\section{Method 3 : Parametric reception using phase modulation}

The third modality proposed to measure nonlinearity in trabecular bone is parametric reception using a phase modulation method.

\subsection{Experimental method}

In this method a low-frequency wave $\left(\mathrm{f}_{L F}=150 \mathrm{~Hz}\right)$ and a high-frequency wave $\left(\mathrm{f}_{H F}=800 \mathrm{kHz}\right)$ are transmitted in the medium in perpendicular directions and cross in the sample immersed in water (figure 10). The LF wave is generated by an $8 \mathrm{~cm}$ diameter circular piston fixed to a shaker while the $\mathrm{HF}$ wave is emitted by a plane transducer. Nonlinear interactions in the water and in the sample generate two parametric waves at the sum and difference of the two primary frequencies $\mathrm{f}_{L F}$ and $\mathrm{f}_{H F}$. If those parametric frequencies belong to the bandwidth of the $\mathrm{HF}$ receiver, this can be interpreted as a modulation of the HF wave phase. The propagation velocity of the HF wave in the medium is modulated by the LF wave due to nonlinearity.

Because of the high primary frequency ratio $\mathrm{f}_{H F} / \mathrm{f}_{L F}$, and if the interaction distance is much less than the LF wavelength, the LF acoustic disturbance appears to be quasi-static for the HF wave. Considering two plane waves interacting along the distance $\mathrm{L}$ in a homogeneous medium, the phase modulation is given by integration of the wave number along the interaction distance :

$$
\phi(t)=\frac{2 \pi f_{H F} L \beta}{c^{2}} v_{L F}(t)
$$

where c, $\beta$ and $v_{L F}$ are the propagation velocity, the classical quadratic nonlinear parameter and the LF acoustic velocity, respectively. $\beta$ is defined as 
$\frac{\rho_{0}}{2}\left(\frac{\partial c^{2}}{\partial p}\right)_{0}$, where $\rho_{0}$ is the density of the medium and $p$ the acoustic pressure. Thus, according to classical nonlinear theory, the phase modulation has the same temporal form as the LF acoustic velocity.

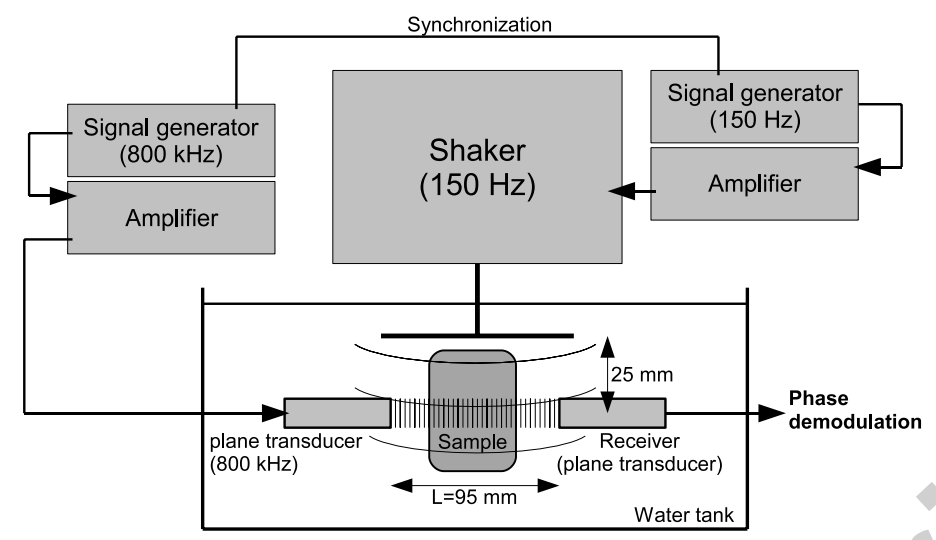

Fig. 10. Experimental configuration for parametric reception.

If we insert a sample (as presented on figure 10) of different density, velocity and nonlinear parameter in a water tank, the phase modulation is now expressed by :

$$
\begin{gathered}
\phi(t)=\frac{2 \pi f_{H F}\left(L-L_{\text {sample }}\right) \beta_{\text {water }}}{c_{\text {water }}^{2}} v_{L F \text { water }}(t) \\
+\frac{2 \pi f_{H F} L_{\text {sample }} \beta_{\text {sample }}}{c_{\text {sample }}^{2}} T_{\text {water-sample }} v_{L F w a t e r}(t),
\end{gathered}
$$

where $T_{\text {water-sample }}$ is the transmission coefficient for acoustic velocity given by :

$$
T_{\text {water-sample }}=\frac{2 Z_{\text {water }}}{Z_{\text {water }}+Z_{\text {sample }}},
$$

where $Z_{\text {water }}$ and $Z_{\text {sample }}$ are the acoustic impedance of water and the sample, respectively. The experiment consisted of measuring the increase in phase modulation as a function of the LF wave amplitude. Repeating the protocol in water (without sample) and with a sample provided the ratio $\beta_{\text {sample }} / \beta_{\text {water }}$. Because the LF pressure amplitude is proportional to the inverse of the distance from the piston, the sample was placed as close as possible to the piston and the HF beam axis was thus $2.5 \mathrm{~cm}$ below the piston. The maximum LF pressure amplitude measured in water, corresponding to a $300 \mathrm{mV}$ peak-peak excitation of the transducer, was $2000 \mathrm{~Pa}$ in the cross-section of the LF and HF beams. The pressure under the LF piston was nearly uniform at $2.5 \mathrm{~cm}$ under the piston, except near the edges. The HF wave was received by a plane transducer after propagation through the sample. The phase modulation amplitude was obtained by numerical analysis. The signal was band-pass filtered around $800 \mathrm{kHz}$ to suppress noise, then the phase of the received signal was 
calculated by Hilbert transform and phase unwrapping. Finally the slope of $\omega t$ was subtracted and the modulation contribution remained. The mean amplitude of the phase modulation was calculated using Fast Fourier Transform analysis. The phase noise level was below $0.5 \mathrm{mrad}$.

\subsection{Results}

By performing the experiment in water, i.e. without a sample, and with a sample placed in the cross-section of the two beams, it is possible to evaluate the nonlinear parameter of the sample. The phase modulation amplitude is reported as a function of the LF wave amplitude on figure 11. We found $\beta=$ 14 for Plexiglas (PMMA), whereas the usual value is 7.5. This over-estimation was probably due to reflections inside the Plexiglas sample. Attenuation was weak (Table 1 ) and the reflection coefficient for acoustic velocity was 0.38 . $\mathrm{Cu}$ mulative effects can occur and enhance the phase modulation. These effects may be negligible in trabecular bone and porous ceramic because of strong attenuation. Considering the bone and ceramic samples as effective media, we found $\beta$ values of 36 and 3400, respectively.

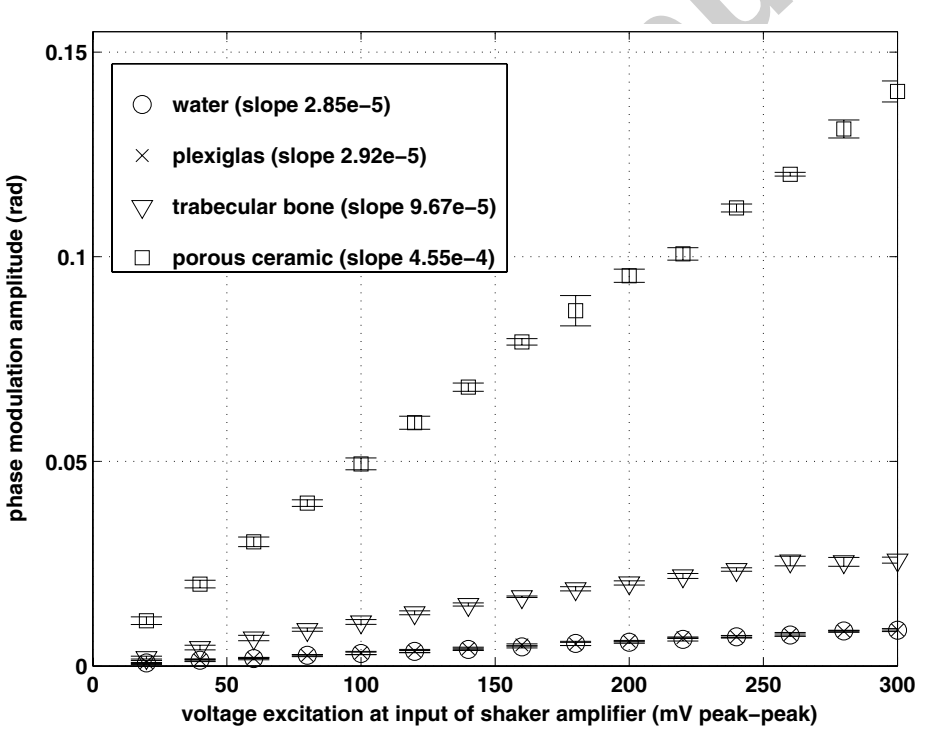

Fig. 11. Results for phase modulation obtained for water (without sample), Plexiglas, trabecular bone and porous ceramic. The noise level was measured around 1 mrad.

As the nonlinear effects happened mainly in the solid phase, the corresponding $\beta$ had to be estimated using the solid phase properties instead of the effective media properties $\left(T_{\text {water-sample }}, c_{\text {sample }}\right.$ and $\left.L_{\text {sample }}\right)$ (see Table 1$)$. We then obtained $\beta$ values of 440 and 24000 for trabecular bone and porous ceramic, respectively. Porous ceramic exhibited a much higher nonlinear param- 
eter than trabecular bone. Bone tissue comprises $70 \%$ calcium hydroxyapatite crystals and $30 \%$ organic collagen fibers (in volume) whereas porous ceramic comprises only industrially made calcium hydroxyapatite crystals. This material used in surgery may not contain microcracks. It is composed of juxtaposed grains (like rocks) of typical size in the order of $1 \mu \mathrm{m}$. For higher LF wave amplitudes (above $2000 \mathrm{~Pa}$ ) phase modulation increased with different nonlinear behaviors that require further investigation.

Table 1

Characteristics of samples and experimental results.

\begin{tabular}{|c|c|c|c|c|}
\hline & water & Plexiglas & trabecular bone & porous ceramic \\
\hline porosity (\%) & & & 90 & 75 \\
\hline solid phase density $\left(\mathrm{kg} / \mathrm{m}^{3}\right)$ & 1000 & 1180 & 1900 & 3100 \\
\hline solid phase velocity $(\mathrm{m} / \mathrm{s})$ & 1480 & 2700 & 3000 & 5500 \\
\hline effective density $\left(\mathrm{kg} / \mathrm{m}^{3}\right)$ & & & 1090 & \\
\hline effective velocity $(\mathrm{m} / \mathrm{s})$ & & & 1650 & 4000 \\
\hline$\beta$ (effective medium) & 2.5 & 14 & 36 & 3400 \\
\hline thickness (mm) & & 24 & 4 & 20 \\
\hline$\beta$ (solid phase only) & & & 440 & 24000 \\
\hline $\begin{array}{l}\text { thickness of solid phase }= \\
\text { thickness } \times \text { porosity }{ }^{1 / 3}(\mathrm{~mm})\end{array}$ & & & 11 & 13 \\
\hline sample attenuation, $400 \mathrm{kHz}(\mathrm{dB} / \mathrm{cm})$ & 0.0003 & 2 & 7 & 18 \\
\hline sample attenuation, $800 \mathrm{kHz}(\mathrm{dB} / \mathrm{cm})$ & 0.0013 & 3 & 20 & 36 \\
\hline sample attenuation, $1.2 \mathrm{MHz}(\mathrm{dB} / \mathrm{cm})$ & 0.003 & 5 & 30 & 50 \\
\hline
\end{tabular}

\section{Discussion and conclusion}

The aim of this preliminary study was to define the best method(s) to measure and scan trabecular bone acoustic nonlinearity. The measurement of second and third harmonic amplitudes as functions of the fundamental amplitude provided valuable information and the second harmonic slope and the level of third harmonic may provide qualitative and/or quantitative information about microdamage. The fundamental frequency must not exceed $400 \mathrm{kHz}$ to limit attenuation and velocity dispersion. Nevertheless, this technique needs fairly large fundamental amplitudes, especially for in vivo application. Soft tissue and the dense cortical shell of the heel bone (which was removed in this study) add an attenuation of 10 to $15 \mathrm{~dB}$ before the wave reaches the trabecular bone area, meaning that three to six times more than the $112 \mathrm{kPa}$ 
used in the present study is necessary. To avoid biological thermal effects, the acoustic pressure amplitude must not exceed 0.5 to $1 \mathrm{MPa}$ for a long $400 \mathrm{kHz}$ burst (tens of milliseconds), although the threshold was in fact not clearly established. Moreover, harmonics are also attenuated by 10 to $15 \mathrm{~dB}$ when propagating through the cortical shell and soft tissue, i.e. from trabecular bone to water surrounding the heel. This means that the fundamental pressure amplitude required (in water before propagating in the heel) would be around $1 \mathrm{MPa}$. Although this configuration is not appropriate for in vivo application, it is a simple experimental set-up for in vitro studies.

The parametric generation configuration presented the same drawback. Resonance effects can be used to enhance the level of the parametric wave (as in the present parametric emission experiment). Nonetheless, evaluation of the gain due to resonance for each point is necessary to interpret two different values measured in two regions of the bone. Perhaps an ad hoc parametric frequency should be chosen for each point of the scan. The maximum variation in ultrasound velocity measured in a heel bone is approximately $300 \mathrm{~m} / \mathrm{s}$ (typical velocities are in the range $1700-2000 \mathrm{~m} / \mathrm{s}$ ). However, we previously reported that in whole heel bone the surrounding cortical surfaces directly influence propagation of the primary wave and provide an image that is more related to relief than to the internal structures [5]. Several effects related to reflection and attenuation on complicated bone shape make the images smoother and more difficult to interpret. However, it is currently difficult to prevent these effects.

In contrast to the other two methods, parametric reception using the phase modulation method does not need a high level of energy. Measurement of the phase modulation at a given LF pressure showed a small standard deviation, but the accuracy and reproducibility of the parametric reception method require evaluation. Moreover, the nonlinear behaviors of the increase in the phase modulation need to be investigated for higher LF pressures. Measurement of the second-order phase modulation induced by parametric frequencies $\mathrm{f}_{H F} \pm 2 \mathrm{f}_{L F}$ must also be studied. The amplitude of the second-order phase modulation is proportional to the hysteretic nonlinear parameter $\alpha$ [26] and may provide different information on bone microdamage. Impact can be used instead of a LF wave and this was tested for nondestructive evaluation applications [25]. Further study is also needed to establish which of the two methods is the most accurate. Parametric reception using phase modulation is thus the best configuration to measure in vivo bone acoustic nonlinearity in order to assess crack density. Phase modulation will be measured using focused rather than plane HF transducers to study spatial variations. The relationship between levels of acoustic nonlinearity and crack density needs to be validated by histological analysis. 


\section{References}

[1] P. Ammann and R. Rizzoli, Bone strength and its determinants, Osteoporos. Int., 14(3) (2003) 13-18.

[2] D. B. Burr, M. R. Forwood, D. P. Fyhrie, R. B. Martin, M. B. Schaffler and C. H. Turner, Bone microdamage and skeletal fragility in osteoporotic and stress fractures, J. Bone Miner. Res., 12(1) (1997) 6-15.

[3] D. B. Burr and T. Stafford, Validity of the bulk-staining technique to separate artifactual from in vivo bone microdamage, Clin. Orthoped. Relat. Res., 260 (1990) 305-308.

[4] S. Callé, J. P. Remenieras, O. Bou Matar and F. Patat, Presence of nonlinear interference effects as a source of low frequency excitation force in vibroacoustography, Ultrasonics, 40 (2002) 873-878.

[5] S. Callé, J. P. Remenieras, O. Bou Matar, M. Defontaine and F. Patat, Application of nonlinear phenomena induced by focused ultrasound to bone imaging, Ultrasound in Med. and Biol., 29 (2003) 465-472.

[6] M. A. Gomez, M. Defontaine, B. Giraudeau, E. Camus, L. Colin, P. Laugier and F. Patat, In vivo performance of a matrix-based quantitative ultrasound imaging device dedicated to calcaneus investigation, Ultrasound in Med. and Biol., 28(10) (2002) 1285-1293.

[7] M. Fatemi and J. F. Greenleaf, Ultrasound-stimulated vibro-acoustic spectrography, Science, 280 (1998) 82-85.

[8] N. L. Fazzalari, J. S. Kuliwaba and M. R. Forwood, Cancellous Bone Microdamage in the Proximal Femur: Influence of Age and Osteoarthritis on Damage Morphology and Regional Distribution, Bone, 31(6) (2002) 697-702.

[9] R. A. Guyer and P. A. Johnson, Nonlinear mesoscopic elasticity : evidence for a new class of materials, Physics Today, 52 (1999) 30-35.

[10] R. A. Guyer, K. R. McCall and G. N. Boitnott, Hysteresis, discrete memory and nonlinear wave propagation in rock: a new paradigm, Phys. Rev. Lett., 74 (1995) 3491-3494.

[11] Y. Hamiel, O. Katz, V. Lyakhovsky, Z. Reches and Y. Fialko, Stable and unstable damage evolution in rocks with implications to fracturing of granite, Geophys. J. Int., 167 (2006) 1005-1016.

[12] D. L. Johnson, S. Kostek and A. N. Norris, Nonlinear tube waves, J. Acoust. Soc. Am., 96 (1994) 1829-1843.

[13] S. Mori, R. Harruff, W. Ambrosius and D. B. Burr, Trabecular bone volume and microdamage accumulation in the femoral heads of women with and without femoral neck fractures, Bone, 21(6) (1997) 521-526. 
[14] M. Muller, Mise au point d'une technique ultrasonore d'acoustique non linaire pour l'etude de l'endommagement osseux (Development of a nonlinear ultrasound technique to assess bone damage), PhD University Paris VIII (2006).

[15] M. Muller, A. Sutin, R. Guyer, M. Talmant, P. Laugier and P. A. Johnson, Nonlinear resonant ultrasound spectroscopy (NRUS) applied to damage assessment in bone, J. Acoust. Soc. Am., 118(6) (2005) 3946-3952.

[16] V. E. Nazarov and A. M. Sutin, Nonlinear elastic constants of solids with cracks, J. Acoust. Soc. Am., 102 (1997) 3349-3354.

[17] D. A. Nelson and D. T. Blackstock, Harmonic generation, propagation and attenuation for finite-amplitude tones in an air-filled porous material, Frontiers of Nonlinear Acoustics : Proceedings of 12th ISNA (1990).

[18] P. H. Nicholson and M. L. Bouxsein, Quantitative ultrasound does not reflect mechanically induced damage in human cancellous bone, J. Bone. Miner. Res., 15(12) (2000) 2467-72.

[19] R. J. O'Connell and B. Budiansky, Seismic velocities in dry and saturated cracked solids, J. Geophys. Res., 79 (1974) 5412-5426.

[20] L. Ostrovsky and P. A. Johnson, Dynamic nonlinear elasticity in geomaterials, Rivista del Nuovo Cimento, 24 (2001) 1-46.

[21] J. P. Remenieras, O. Bou Matar, F. Ossant and F. Patat, A simple calculation approach for the parametric sound field generated by a focused annular array. Application to vibro-acoustography, Halliwell and Wells P, ed., New-York: Kluwer Academic / Plenum Publishers, Acoustical Imaging, 25 (2000) 145-150.

[22] M. B. Schaffler, K. Choi and C. Milgrom, Aging and Matrix Microdamage Accumulation in Human Compact Bone, Bone, 17(6) (1995) 521-525.

[23] O. S. Sobelman, J. C. Gibeling, S. M. Stover, S. J. Hazelwood, O. C. Yeh, D. R. Shelton and R. B. Martin, Do microcracks decrease or increase fatigue resistance in cortical bone?, J. Biomech., 37(9) (2004) 1295-1303.

[24] D. M. Donskoy and A. Sutin, Nonlinear acoustic parameter of trabecular bone, J. Acoust. Soc. Am., 102 (1997) 3155.

[25] K. E. A. Van Den Abeele, A. Sutin, J. Carmeliet and P. A. Johnson, Microdamage diagnostics using nonlinear elastic wave spectroscopy (NEWS), NDT \& E Int., 34(4) (2001) 239-248.

[26] K. E. A. Van Den Abeele, P. A. Johnson and A. Sutin, Nonlinear Elastic Wave Spectroscopy (NEWS) Techniques to Discern Material Damage, Part I: Nonlinear Wave Modulation Spectroscopy (NWMS), Res. Nondestr. Eval., 12 (2000) 17-30.

[27] K. E. A. Van Den Abeele, J. Carmeliet, J. Ten Cate and P. A. Johnson, Nonlinear Elastic Wave Spectroscopy (NEWS) Techniques to Discern Material Damage, Part II: Single-Mode Nonlinear Resonance Acoustic Spectroscopy, Res. Nondestr. Eval., 12(1) (2000) 31-42. 
[28] K. A. Wear, Measurements of phase velocity and group velocity in human calcaneus, Ultrasound in Med. and Biol., 26(4) (2000) 641-646.

[29] V. Zaitsev, V. Nazarov, V. Gusev and B. Castagnede, Novel nonlinearmodulation acoustic technique for crack detection, NDT\&E Int., 39 (2006) 184194.

[30] P. Zioupos, Ageing human bone: factors affecting its biomechanical properties and the role of collagen, J. Biomater. Appl., 15(3) (2001) 187-229.

[31] P. Zioupos, Accumulation of in vivo fatigue microdamage and its relation to biomechanical properties in ageing human cortical bone Journal of Microscopy, 201(2) (2001) 270-278. 